\title{
Effect of Special Finishes on the Functional Properties of Cotton Fabrics
}

\author{
Kawser Parveen Chowdhury \\ Department of Wet Process Engineering, Faculty of Textile Chemical Engineering, Bangladesh University of Textiles, Dhaka, \\ Bangladesh \\ Email: kpc.urmi@butex.edu.bd
}

How to cite this paper: Chowdhury, K.P. (2018) Effect of Special Finishes on the Functional Properties of Cotton Fabrics. Journal of Textile Science and Technology, 4, 49-66.

https://doi.org/10.4236/jtst.2018.42003

Received: March 9, 2018

Accepted: May 6, 2018

Published: May 9, 2018

Copyright $\odot 2018$ by author and Scientific Research Publishing Inc. This work is licensed under the Creative Commons Attribution-NonCommercial International License (CC BY-NC 4.0). http://creativecommons.org/licenses/by-nc/4.0/ cc) (i) (8) Open Access

\begin{abstract}
This study demonstrated that the changes in functional properties of both woven and knit cotton fabrics were determined to evaluate the performance of different special finishes. Here, $100 \%$ cotton fabrics were treated with different types of finishing chemicals at different formulations. To assess the performance of different finishes on fabric properties, GSM, bursting strength, tensile strength, dimensional stability, absorbency, crease recovery angle with ASTM and AATCC methods were done. The results showed that the functional properties of the finished cotton fabrics both woven and knit depend to a great extent on the type of finish.
\end{abstract}

\section{Keywords}

Water Repellent Finish, Wrinkle Free Finish, Soft Finish, Paper Touch Finish, Knit Fabric, Woven Fabric, Performance

\section{Introduction}

Cotton is a versatile fibre with outstanding quality regards comfort ability. Softness, water repellency, wrinkle free, silky soft, antistatic property are some of the most common functional properties that are needed for protective clothing without affecting the comfort of the cotton fabric. Generally different special types of finishes are applied on cotton fabric to impart various functional properties. Here ninth types of special finishes are applied on both cotton woven and knit fabrics.

The first finish is antistatic finish with siligen softener SIH (Figure 1) which is a modified polysiloxane. It provides excellent hydrophilic, soft handle and antistatic property for cotton and their blends with synthetic fibres. 


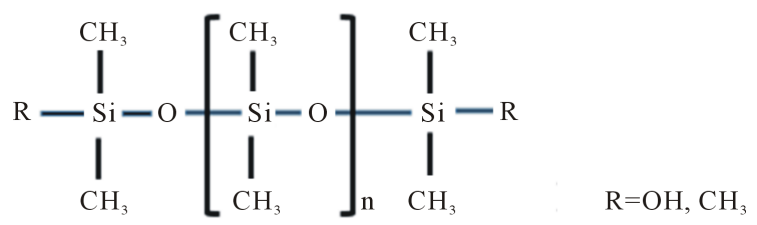

Figure 1. Chemical structure of dimethyl polysiloxane [1].

The second finish is polyurethane finish with perapret additive PU New (Figure 2) which is a film forming, anionic polyurethane dispersion. It provides a chintz effect on cotton fabric. Higher application of this finishing agent gives a fuller handle.

The third and forth finishes are soft finishes named as silicon (Micro) and silicon (Macro) both of which are generally polysiloxane derivatives of low molecular weight (Figure 3). They are water insoluble and must be applied on fabrics after dissolution in organic solvents, or in the form of disperse products. They create a lubricating and moderately waterproof film on the surface and give fabrics a velvety silky handfeel. They are generally used for soft finish and silky soft finishes to impart soft handle on fabric.

Both silicon (Micro) and silicon (Macro) finishes are done by using siligen softener SIE (Figure 3 ) which is an amino functional polysiloxane, provides very smooth and soft handle for cotton and their blends with synthetic fibres.

The fifth finish is polyethylene finish with perapret additive PEP (Figure 4) agent, and what is used in this experiment is polyethylene waxes (polyethylene).

The sixth finish is water repellent finish which is the ability of a textile material to resist wetting [2] [3]. There has been a market increase in the commercial use of fluorocarbons in recent years, particularly to impart water repellency to cotton [4]-[10]. Some existing fluorocarbons are made with C8 carbon backbone chains that can release perfluoro octane sulfonate (PFOS) and perfluoro octanoic acid (PFOA) and other toxic and hazardous materials [11]. Here the water repellent agent is fluorocarbon. The final polymer, when applied to a fibre, should form a structure that presents a dense $\mathrm{CF}_{3}$ outer surface for maximum repellency. A typical structure is shown in below Figure 5. The length of the perfluorinated side chains should be about 8 carbons. Co-monomers are X, Y, for example are stearyl- or lauryl-meth-acrylate, butyl-acrylate, methylol- or epoxy-functional acrylates.

All water repellent chemicals are usually available as water emulsions and are used to fabric by the pad-dry-cure method with a curing temperature around $150-170^{\circ} \mathrm{C}$ for a couple of minutes [6] [7] [8] [9].

Cotton is intermolecularly linked by many hydroxyl groups in two different regions: one is crystalline region and another is amorphous region. Cellulose chains are closed packed in crystalline region which limits their mobility and accessibility of molecules, whereas they are temporarily held with weak hydrogen bonds in the amorphous region. When a force is applied onto the fibre during wear and laundering process, hydrogen bonds are easily broken and slippage 
<smiles>[R]NC(=O)N(C)CO[R]</smiles>

Figure 2. Alkyl urethane structure [1].

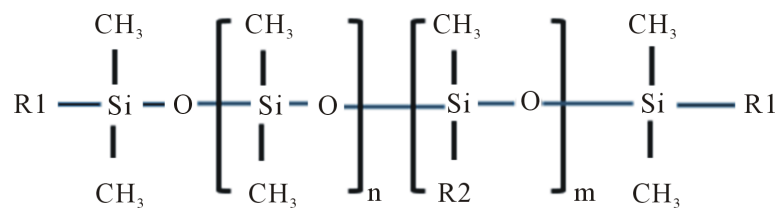

$\mathrm{R} 1=\mathrm{OH}, \mathrm{CH}_{3}$

$\mathrm{R} 2=\left(\mathrm{CH}_{2}\right)_{3}-\mathrm{NH}-\left(\mathrm{CH}_{2}\right)_{2}-\mathrm{NH}_{2},\left(\mathrm{CH}_{2}\right)_{3}-\mathrm{NH}_{2}$

Figure 3. Chemical structure of dimethyl polysiloxane containing amino group [1].

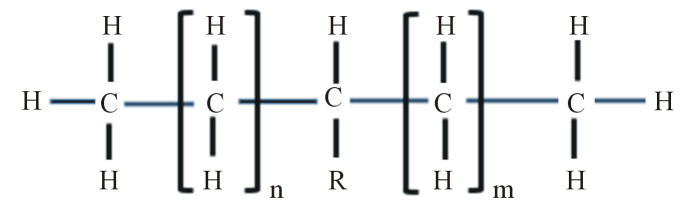

$\mathrm{R}=\mathrm{COOH}$

Figure 4. Chemical structure of polyethylene waxes [1].

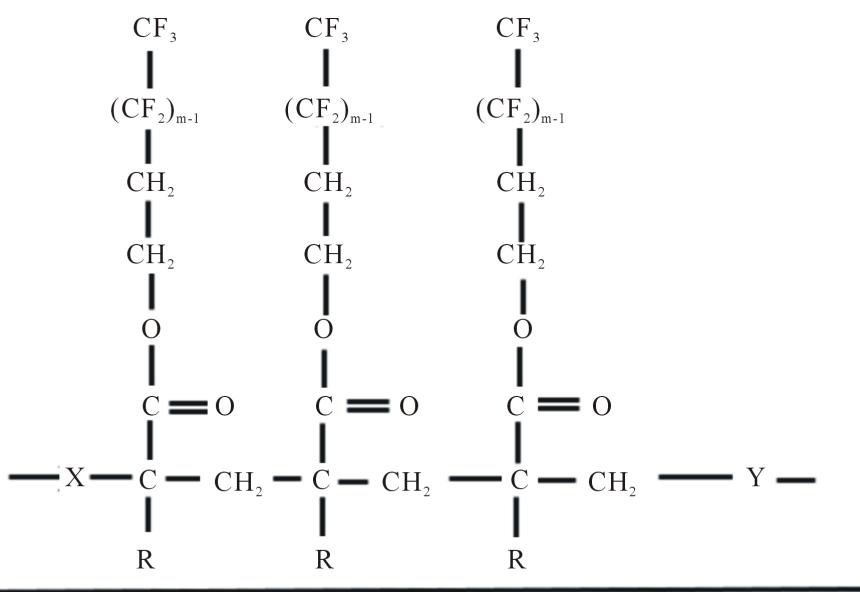

A

Figure 5. Fluorocarbon repellent on fibre surface. $\mathrm{M}=8 . \mathrm{X}$ and $\mathrm{Y}$ are co-monomers, mainly stearylates. $\mathrm{R}=\mathrm{H}$ or $\mathrm{CH}_{3}$ (polyacrylic or polymethacrylic acid esters). $\mathrm{A}$ is the fibre surface [1].

occurred between the cellulose chains. When slippage occurs, new hydrogen bonds formed at different location and tend to maintain the fibre in wrinkled or bent state. For this reason, wrinkles are occurred in fabric appearance. Moreover, as cotton fibre is hydrophilic, the absorption of water breaks the hydrogen bonds and allow the fibre to shrink by preventing the chains from returning to their original positions, even after the applied forces or water are removed. Due 
to this reason, cotton fibre is easily wrinkled and shrinked after laundering which is one of the main drawback of cotton fibre that make them inconvenience for users [12] [13] [14] [15] [16]. Introduce of wrinkle is mainly depends on some factors of fibre, yarn and fabric characteristics and finishing process such as fibre type, bending performance of fibre, fibre diameter, type and number of yarn twist, fabric density, fabric construction and thickness [16].

To overcome wrinkles, cotton fabrics are treated with chemicals by applying a special type of finish named wrinkle resistant finish by crosslinking of hydroxyl groups of cellulose. Various types of resins have been developed to improve wrinkle or crease resistant of cotton fabrics. Dimethylol dihydroxy ethylene urea (DMDHEU) resin was mainly used among different types of resins as it is commercially widely accepted in industry due to its superior crease recovery property and low cost [17] [18].

The seventh finish is wrinkle free finish by using fixapret ECO (Figure 6) which is a modified cross linker based on DMDHEU, methanol and DEG. It contains extremely low levels of uncombined formaldehyde. The chemical reaction of fixapret with cellulose is given in Figure 7.

The eighth finish is paper touch finish which is done by using perapret VA finishing agent. It is a nonionic dispersion of polyvinyl acetate. It is generally used for filling and stiffening effects on cotton fabrics and their blends with synthetic fibres. High add on of this agent allow a very stiff handle.

The ninth finish is silky soft finish which is done by perapret F-PEB finishing agent which is a secondary polyethylene dispersion.

In the literature, a considerable number of studies on different finishing chemicals, their application procedure, methods for upgrading the durability

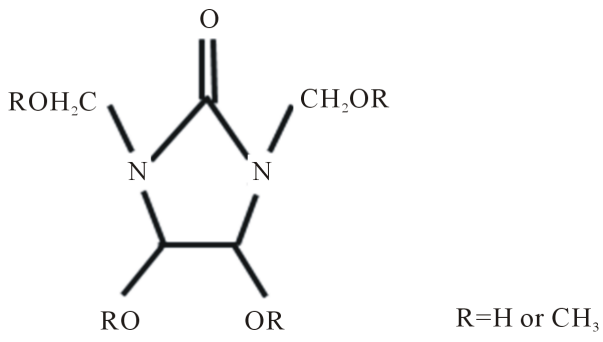

Figure 6. Chemical formula of Fixapret CL [1].

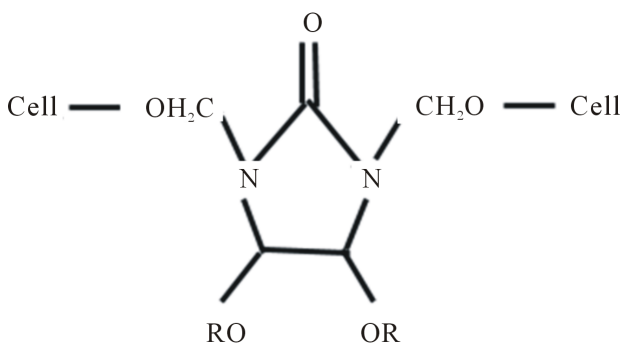

Figure 7. Di-ether crosslinking between DMDHEU and cellulose polymers; cell $=$ cellulose $[19]$. 
and their wash fastness and curing temperature have been reported [20] [21] [22]. There are almost insufficient work on the performance of different finishes and their effects on both woven and knit cotton fabric's properties. The purpose of this study is to observe and evaluate the performance of different finish effects for both woven and knit fabric properties after finishing.

\section{Materials and Methods}

\subsection{Materials}

$100 \%$ cotton one woven and one knit fabrics (scoured and bleached) are used for this experiment to apply different types of finishes by pad-dry-cure method. The woven fabric is collected from local shops and knit fabric is supplied by Essential Clothing Ltd., Gazipur, Bangladesh. Woven fabric has plain weave structure with 110 GSM and knit fabric is single jersey with 160 GSM. Siligen softener SIH (nonionic antistatic finishing agent based modified polysiloxane, BASF), Kieralon wash JET-B Conc (nonionic surfactant with wetting and emulsifying property, BASF), Perapret additive PU New (nonionic polyurethane based finishing agent, BASF), Siligen Softener SIS (one softener is silicon micro emulsion and another softener is silicon nano emulsion, nonionic, BASF), Perapret Additive PEP (nonionic polyethylene wax based finishing agent, BASF), Nuva TTC (fluorocarbon based water repellent finishing agent, weakly cationic, dispersion of fluorine compound, Clariant), Fixapret F-ECO (modified dimethylol dihydroxy ethylene urea, cationic, BASF), Fixapret Catalyst F-M (a synergistic mixture of metal salts used for as a catalyst to initiate crosslinking reaction with cross linker, BASF), Perapret F-PEB (a secondary polyethylene dispersion, BASF), Siligen Softener SIO (softener, BASF), Perapret Stiffener VA New (weakly cationic poly vinylacetate-based polymer dispersion, BASF), Siligen Softener SIE (nonionic, amino functional polysiloxane, BASF) are of commercial grade.

\subsection{Methods}

Supplied fabrics are then processed with different types of finishing chemicals to investigate the effect of finishes on other physical properties of the fabrics.

\subsubsection{Antistatic Finish}

Supplied fabrics are then processed with different types of finishing chemicals to investigate the effPortions of woven and knit fabrics (scoured and bleached) are padded using Siligen softener SIH (30 g//L) and Kieralon wash JET-B Conc. (2 $\mathrm{g} / \mathrm{L})$ to a wet pick up of $80 \%$ followed by drying $120^{\circ} \mathrm{C}$ for $2 \mathrm{~min}$.

\subsubsection{Polyurethane Finish}

Portions of woven and knit fabrics (scoured and bleached) are padded using Perapret additive PU New (30 g/L) along with Kieralon wash JET-B Conc. (2 g/L) to a wet pick up of $80 \%$ followed by drying $120^{\circ} \mathrm{C}$ for 2 min and curing at $150^{\circ} \mathrm{C}$ for $5 \mathrm{~min}$. 


\subsubsection{Silicon Finish (Macro and Micro)}

Siligen Softener SIS (one is in micro level (particle size $80 \mathrm{~nm}$ ) and another is in macro level (particle size $120 \mathrm{~nm}$ )) is used on cotton fabric in this experiment. Fabrics are finished with Siligen Softener SIS (30 g/L) and Kieralon JET-B Conc. $(2 \mathrm{~g} / \mathrm{L})$ by padding at $80 \%$ pick up and followed by drying at $120^{\circ} \mathrm{C}$ for $2 \mathrm{~min}$.

\subsubsection{Polyethylene Finish}

Fabrics are treated with Perapret Additive PEP (30 g/L) and Kieralon JET-B Conc. $(2 \mathrm{~g} / \mathrm{L})$ by using pad ( $80 \%$ pick up) and dry $\left(120^{\circ} \mathrm{C}\right.$ for $\left.2 \mathrm{~min}\right)$ process on both cotton woven and knit fabrics in this experiment.

\subsubsection{Water Repellent Finish (Fluorocarbon)}

Fabrics are treated with Nuva TTC $(30 \mathrm{~g} / \mathrm{L})$ and Acetic acid $(2 \mathrm{ml} / \mathrm{L})$ by using pad ( $80 \%$ pick up), dry $\left(120^{\circ} \mathrm{C}\right.$ for $\left.2 \mathrm{~min}\right)$ and cure process $\left(150^{\circ} \mathrm{C}\right.$ for $\left.5 \mathrm{~min}\right)$ on both cotton woven and knit fabrics in this experiment.

\subsubsection{Wrinkle Free Finish (Fixapret F-ECO)}

Fixapret F-ECO (20 g/L), Fixapret Catalyst F-M (6 g/L), Perapret F-PEB (3 g/L) and Siligen Softener SIO $((3 \mathrm{~g} / \mathrm{L})$ are used on cotton fabric in this experiment. Fabrics are finished with above chemicals by padding at $80 \%$ pick up, followed by drying at $120^{\circ} \mathrm{C}$ for $2 \mathrm{~min}$ and curing at $150^{\circ} \mathrm{C}$ for $5 \mathrm{~min}$.

\subsubsection{Paper Touch Finish}

Perapret Stiffener VA New (20 g/L), Siligen Softener SIE (10 g/L) and Kieralon wash JET-B Conc. ( $2 \mathrm{~g} / \mathrm{L})$ are used on cotton fabrics in this experiment. Fabrics are finished with above chemicals by padding at $80 \%$ pick up, followed by drying at $120^{\circ} \mathrm{C}$ for $2 \mathrm{~min}$ and curing at $150^{\circ} \mathrm{C}$ for $5 \mathrm{~min}$.

\subsubsection{Silky Soft Finish}

Portions of fabrics are treated by Perapret F-PEB (20 g/L), Siligen Softener SIE $(10 \mathrm{~g} / \mathrm{L})$ and Kieralon wash JET-B Conc. $(2 \mathrm{~g} / \mathrm{L})$ in this experiment. Fabrics are finished with above chemicals by padding at $80 \%$ pick up, followed by drying at $120^{\circ} \mathrm{C}$ for $2 \mathrm{~min}$ and curing at $150^{\circ} \mathrm{C}$ for $5 \mathrm{~min}$.

Both woven and knit fabrics are treated with a general bath condition. The process parameters are adopted as recommended by the supplier. Pad-Mangle machine is used for padding with $2.5 \mathrm{~m} / \mathrm{min}$ fabric speed and $2.8 \mathrm{~kg} / \mathrm{cm}^{2}$ padding pressure. Fine oven machine is used for drying. SDL Mini-Dryer Steamer is used for curing.

\subsection{Fabric Testing}

Each sample is tested in the standard atmosphere, $25 \pm 2{ }^{\circ} \mathrm{C}$ temperature and $65 \% \mathrm{RH}$ after conditioning $24 \mathrm{hrs}$.

\subsubsection{GSM Test}

It is done by GSM cutter from James H. Heal \& Co. Ltd. Halifax, England according to ASTM (D 3776-79) method. 


\subsubsection{Drop Absorbency Time}

It is determined according to AATCC (79-1992) method.

\subsubsection{Tensile Strength Test}

It is done for woven fabric according to ASTM (D 5045) method by Good brand fabric strength tester.

\subsubsection{Bursting Strength Test}

It is done by Trust burst tester according to ASTM (D 3786-87) method.

\subsubsection{Shrinkage Percentage}

It is done according to AATCC 96-1997 method.

\subsubsection{Crease Recovery Angle}

It is done by Shirley crease recovery tester according to AATCC 66-2003 method and is taken the average values of warp and weft results.

\subsubsection{Spray Rating Test}

Spray rating tests are done by Spray Rating Tester by James H. Heal \& Co. Ltd. Halifax, England. AATCC 22-2001 test method is used to evaluate the water repellency of the fabric. Where 100 grade of surface resistance rating expresses that there will be no sticking or wetting of the upper fabric surface, 90 grade rating expresses that there will be only slight random wetting on the upper fabric surface, 80 grade means there will be wetting at the spray points on the upper surface of fabric while a fabric with complete wetting was assigned a 0 rating.

\section{Result and Discussion}

Since the main task of the present study is to observe the effect of different finishing on cotton both woven and knit fabrics, a wide range of finishing formulations have been used and evaluated on the basis of the performance of treated cotton fabric. Nine different finishes are chosen in this work and are applied on plain weave woven and single jersey knit fabrics. Anti-Static finish is expressed as A static, Polyurethane finish is expressed as PU, Silicon (Micro) finish is expressed as Sil (Mic), Silicon (Macro) finish is expressed as Sil (Mac), Polyethylene finish is expressed as PE, Water repellent is expressed as W R, Wrinkle Free is expressed as W F, Paper touch is expressed P touch, Silky soft finish is expressed as Silky throughout the results. All finishes are done by using $32 \mathrm{~g} / \mathrm{L}$ solutions to avoid the effects of add on variation. Results obtained along with their appropriate discussions are as follows.

\subsection{Change in Strength}

Tensile strength plays a vital role after different finishes. It was done according to ASTM (D 5045) method to evaluate the treated woven fabric's tensile strength. In the chart (Table 1, Figure 8 and Figure 9) the tensile strength of the cotton woven fabric shows more for warp than weft due to yarn density and yarn quality and after different finishing process, slight deterioration occurs for both 
Table 1. Tensile strength of woven fabric treated with different finishing agent.

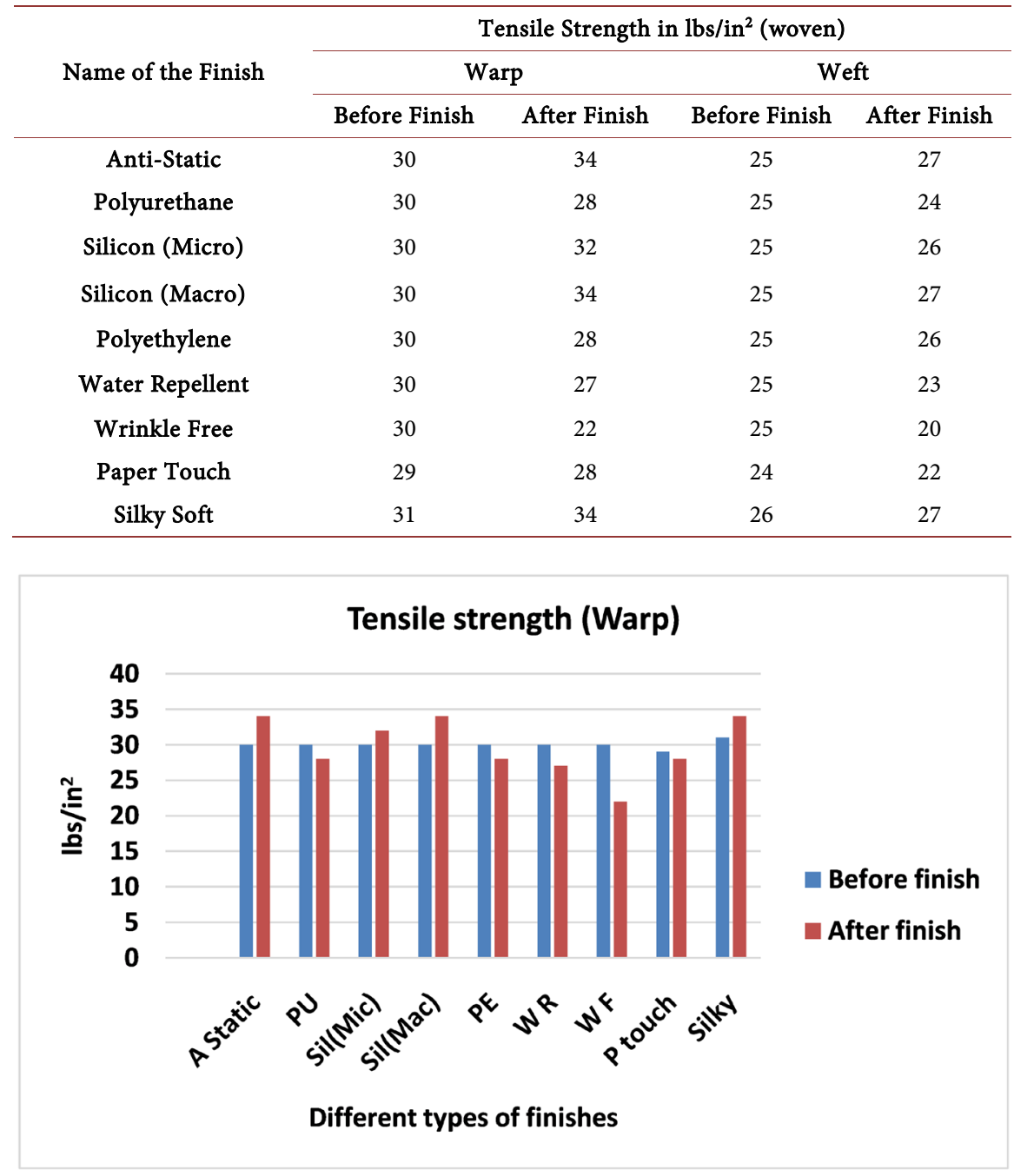

Figure 8. Tensile strength of woven fabric (warp) treated with different finishing agent.

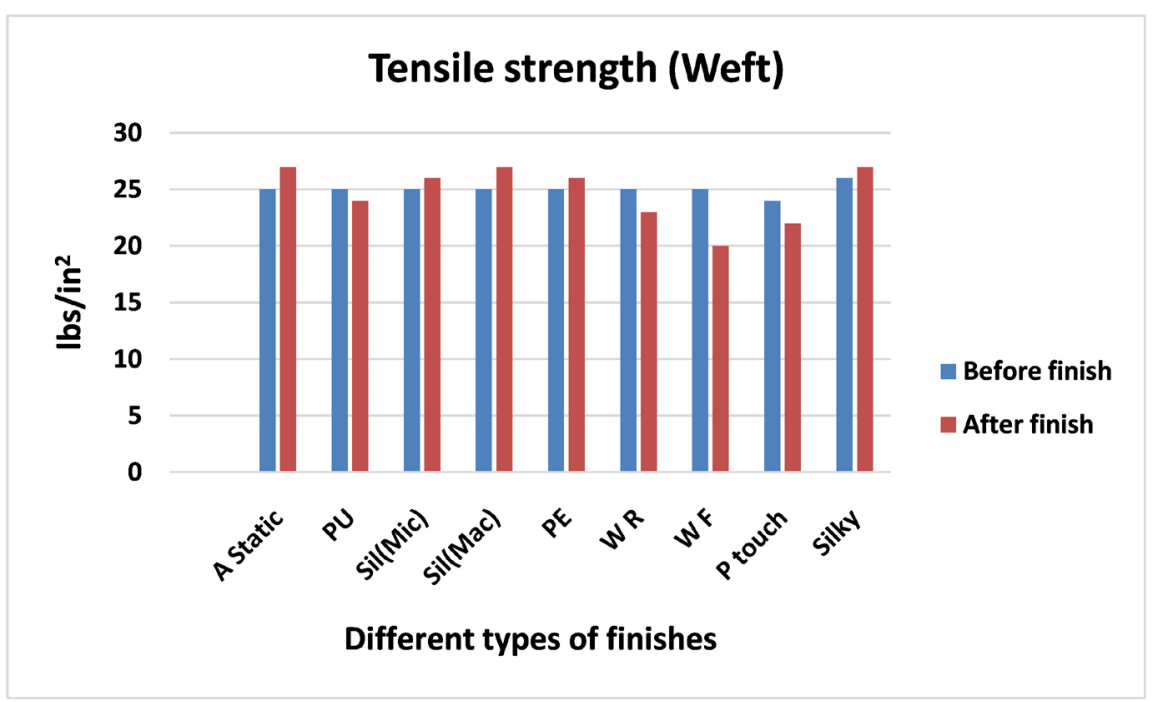

Figure 9. Tensile strength of woven fabric (weft) treated with different finishing agent. 
warp and weft except soft finishes. Soft finishes make fabric flexible and for this reason tensile strength is increased. In wrinkle free finish, tensile strength decreases and it is obviously taken into account as resin crosslinks in amorphous region of cellulose leading lower flexibility and harsh handfeel, though it is reasonable for the fabric to go for the next proceedings.

In all water repellent finishes, tensile strength reduces as resin crosslinks in acetic condition within the amorphous region of cellulose leading lower flexibility and harsh handfeel. For soft, silky soft and antistatic finishes, tensile strength increases due to a direct consequence of deposition and formation of a waxy thin film onto or within the fibre. Paper touch finish make fabric stiff and probably because of that reason tensile strength decrease.

But in the chart (Table 2, Figure 10) the bursting strength of the cotton knit single jersey (S/J) fabric shows slight deterioration occurs after wrinkle free finish and it is obviously taken into account as resin crosslinks in amorphous region of cellulose leading lower flexibility and harsh handfeel. From the chart, it is clear that after water repellent finish the fabric strength is decreased and it's reasonable.

The bursting strength of knit fabric is done according to ASTM (D 3786-87) method and found bursting strength is reduced for water repellent and wrinkle free finishes, because of effect of the cellulosic fiber during cross linking process. When the water repellent and wrinkle free 'chemicals form cross link with the cotton free $\mathrm{O}-\mathrm{H}$ group in the amorphous region, it makes stiff of the fabric and moreover, cross linking reaction is done mainly in acidic condition which are also responsible for the loss of the fabric's bursting strength. Paper touch finish make fabric stiff and for that consequence, reduce bursting strength. For soft (Sil (Mac), Sil (Mic)), silky soft and antistatic finishes, tensile strength increases due to a direct consequence of deposition and formation of a waxy thin film onto or within the fibre.

\subsection{Change of GSM}

The GSM tests are done according to ASTM D 3776-79 method for all finished fabrics. The variation of GSM of woven fabric after finishing has given in below Table 3 and Figure 11. After chemical implementation of both woven and S/J fabrics with various finishing formulations, GSM has increased because chemical has covered up all the pores of the fabric and a chemical coating is created on the fabric. Therefore, the water is not allowed to penetrate into the fabric for water repellent finishes (W R and PE).

Three types of soft finish are used in this work. One is silky soft finish and other two are silicon soft finish in micro and macro level. The variation of untreated and treated fabric's GSM after soft finishing, are depends on the chemicals add on, formation of interfibre and interyarn bonds and the effects of the softeners deposits in and/or onto the structure on the fabric. The variation of GSM of knit fabric after finishing has given in below Table 4 and Figure 12. 
Table 2. Bursting strength of single jersey $(\mathrm{S} / \mathrm{J})$ fabric treated with different finishing agent.

\begin{tabular}{ccc}
\hline \multirow{2}{*}{ Name of the Finish } & \multicolumn{2}{c}{ Bursting Strength (kpa) } \\
\cline { 2 - 3 } & Before Finish & After Finish \\
\hline Anti-Static & 310 & 316 \\
Polyurethane & 310 & 298 \\
Silicon (Micro) & 310 & 324 \\
Silicon (Macro) & 310 & 327 \\
Polyethylene & 310 & 296 \\
Water Repellent & 310 & 291 \\
Wrinkle Free & 310 & 283 \\
Paper Touch & 310 & 302 \\
Silky Soft & 310 & 324 \\
\hline
\end{tabular}

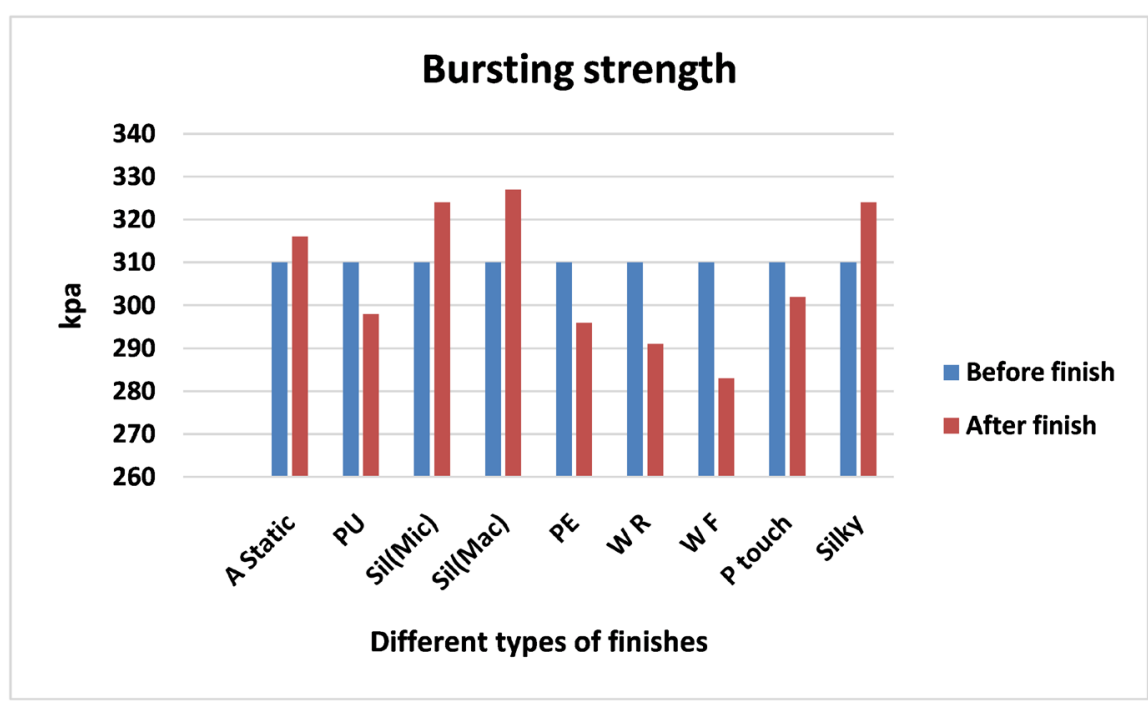

Figure 10. Bursting strength of single jersey $(\mathrm{S} / \mathrm{J})$ fabric treated with different finishing agent.

Table 3. GSM of woven fabrics treated with different finishing agent.

\begin{tabular}{ccc}
\hline \multirow{2}{*}{ Name of the Finish } & \multicolumn{2}{c}{ GSM (Woven) } \\
\cline { 2 - 3 } & Before Finish & After Finish \\
\hline Anti-Static & 110 & 116 \\
Polyurethane & 110 & 118 \\
Silicon (Micro) & 110 & 114 \\
Silicon (Macro) & 110 & 117 \\
Polyethylene & 110 & 116 \\
Water Repellent & 110 & 121 \\
Wrinkle Free & 110 & 123 \\
Paper Touch & 110 & 118 \\
Silky Soft & 110 & 114 \\
\hline
\end{tabular}




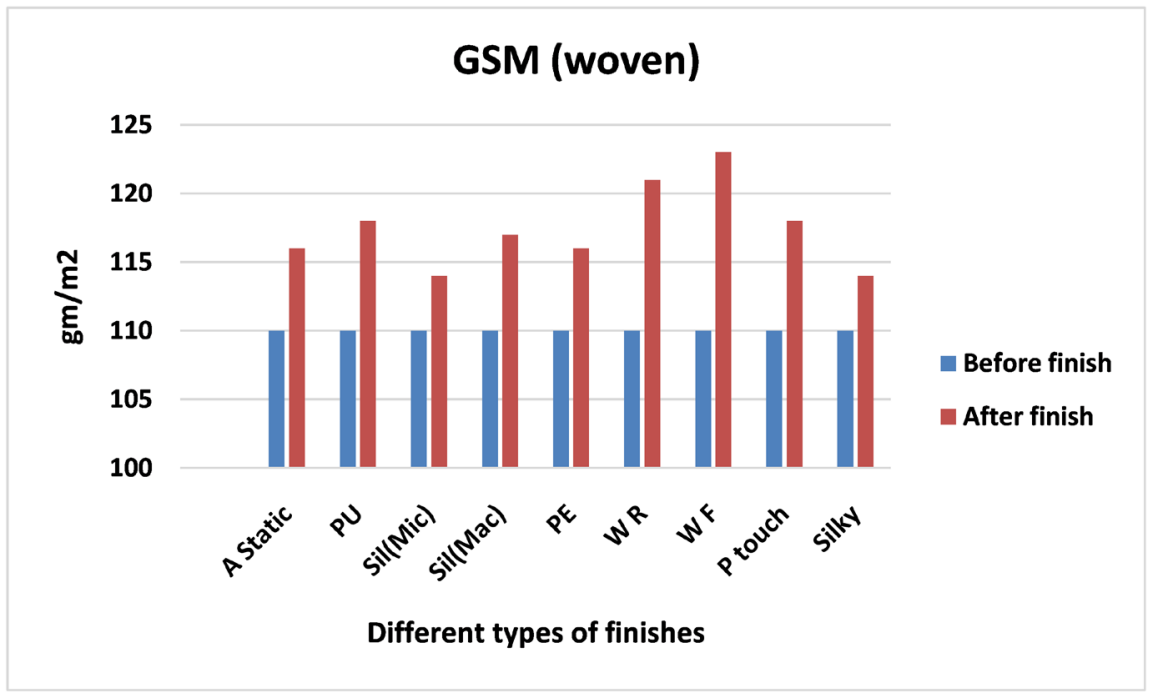

Figure 11. GSM of woven fabrics treated with different finishing agent.

Table 4. GSM of single jersey (S/J) fabric treated with different finishing agent.

\begin{tabular}{ccc}
\hline \multirow{2}{*}{ Name of the Finish } & \multicolumn{2}{c}{ GSM (S/J) } \\
\cline { 2 - 3 } & Before Finish & After Finish \\
\hline Anti-Static & 160 & 165 \\
Polyurethane & 160 & 167 \\
Silicon (Micro) & 160 & 164 \\
Silicon (Macro) & 160 & 167 \\
Polyethylene & 160 & 168 \\
Water Repellent & 160 & 171 \\
Wrinkle Free & 160 & 173 \\
Paper Touch & 160 & 164 \\
Silky Soft & 160 & 166 \\
\hline
\end{tabular}

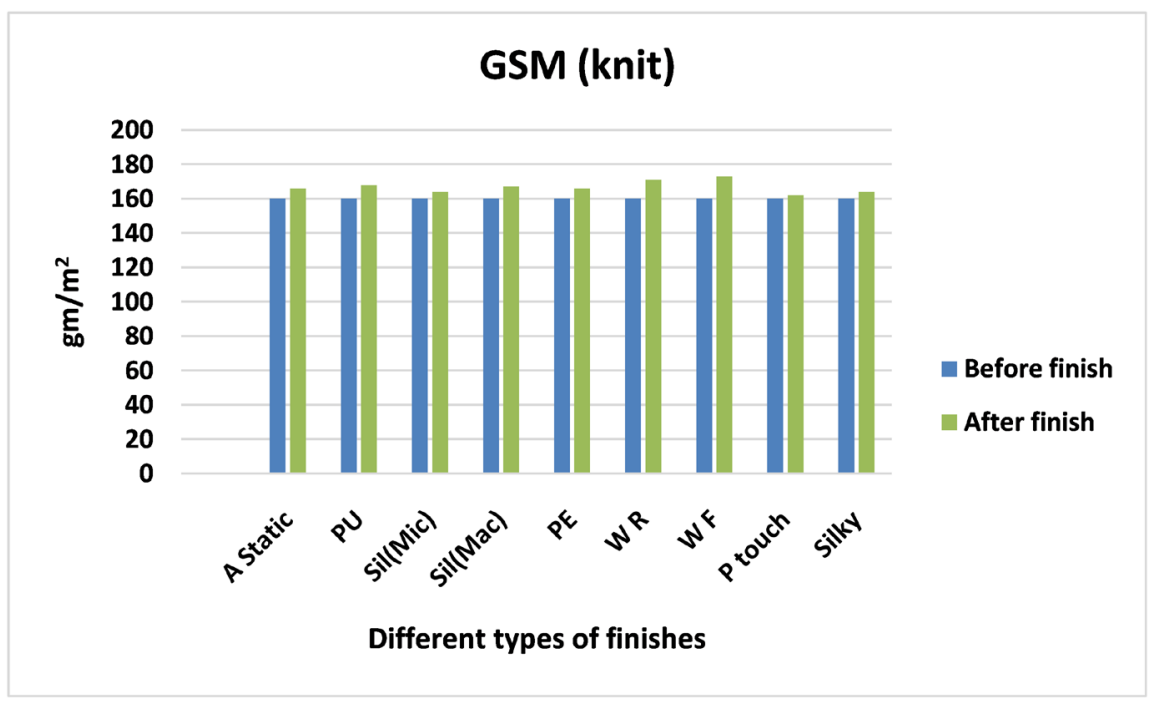

Figure 12. GSM of single jersey (S/J) fabric treated with different finishing agent. 


\subsection{Change in Dimensional Stability}

The dimensional stability or shrinkage tests are done according to AATCC 96-1997 method for all finished fabrics. The variation of shrinkage\% after all finishing process have given in below Table 5 and Figure 13 for woven fabric and Table 6 and Figure 14 for S/J fabric respectively.

After soft finishing dimensional stability are rises due to the mechanical reinforcement by formation of bonds within interfibre and interyarn. Soft finishes reduce the surface roughness by reducing interfibre and interyarn friction. For water repellent finishing, the change in the physico-mechanical and comfort properties of the treated cotton is an improvement of dimensional stability. The improvement of dimensional stability of both knit and woven fabrics are because of the formation of a repellent film on the fibre surface along with a hydrophobic layer around the fibres. After paper touch finish, fabric's stiffness is improved which reduces shrinkage percentage. Moreover, after wrinkle free finish, dimensional stability for both woven and knit fabric is improved due to the crosslinking reaction of the resin.

Table 5. Shrinkage\% of woven fabrics treated with different finishing agent.

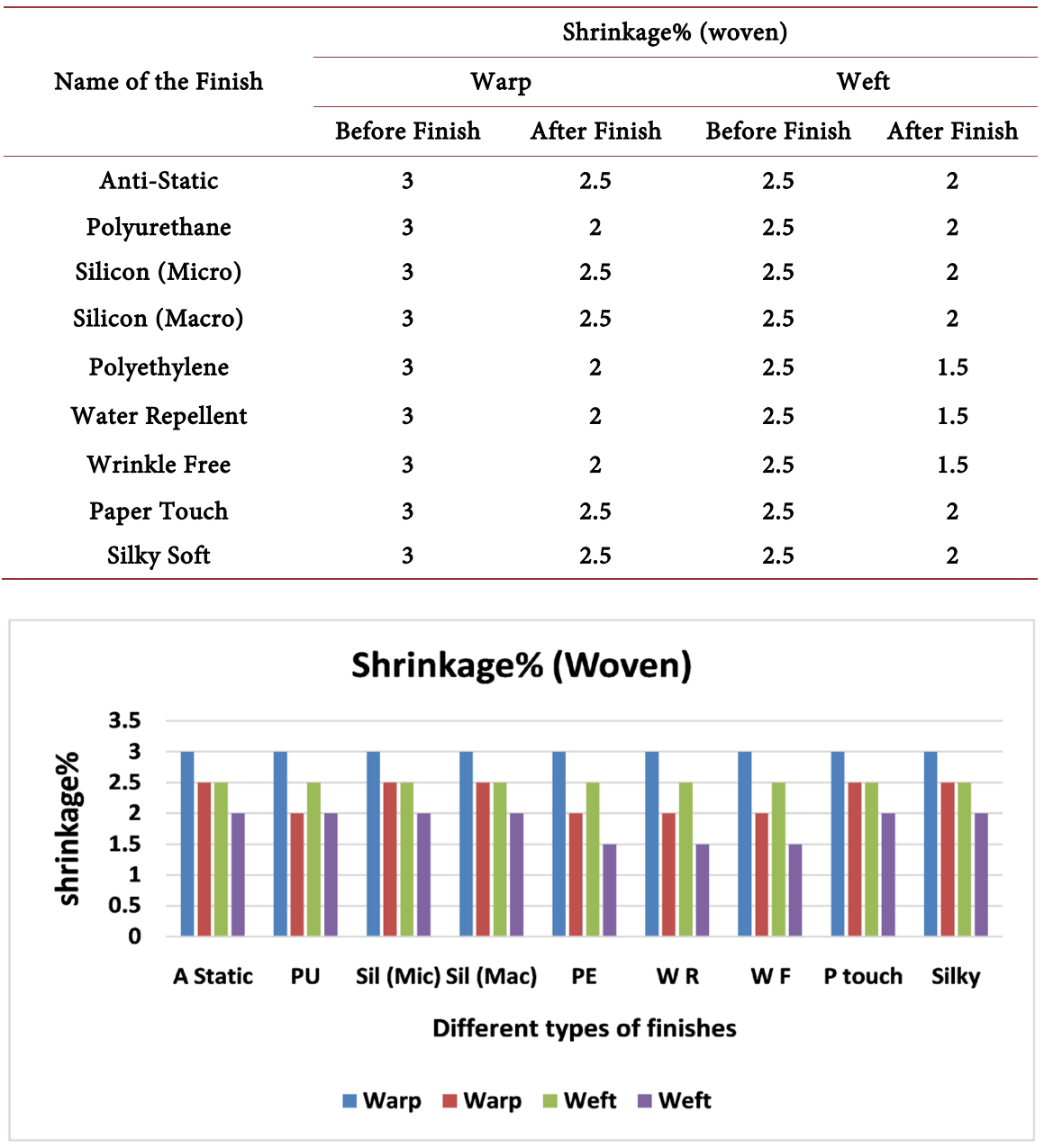

Figure 13. Shrinkage\% of woven fabrics treated with different finishing agent. 
Table 6. Shrinkage\% of single jersey (S/J) fabric treated with different finishing agent.

\begin{tabular}{cccccc}
\hline \multirow{2}{*}{ Name of the Finish } & \multicolumn{4}{c}{ Shrinkage\% (knit) } \\
\cline { 2 - 5 } & \multicolumn{2}{c}{ Wales } & \multicolumn{3}{c}{ Coarse } \\
\cline { 2 - 5 } & Before Finish & After Finish & Before Finish & After Finish \\
\hline Anti-Static & 5 & 4 & 4.5 & 4 \\
Polyurethane & 5 & 4 & 4.5 & 3 \\
Silicon (Micro) & 5 & 3.5 & 4.5 & 3.5 \\
Silicon (Macro) & 5 & 3.5 & 4.5 & 3.5 \\
Polyethylene & 5 & 3 & 4.5 & 3 \\
Water Repellent & 5 & 3.5 & 4.5 & 3 \\
Wrinkle Free & 5 & 3 & 4.5 & 3 \\
Paper Touch & 5 & 3.5 & 4.5 & 3.5 \\
Silky Soft & 5 & 3.5 & 4.5 & 3.5 \\
\hline
\end{tabular}

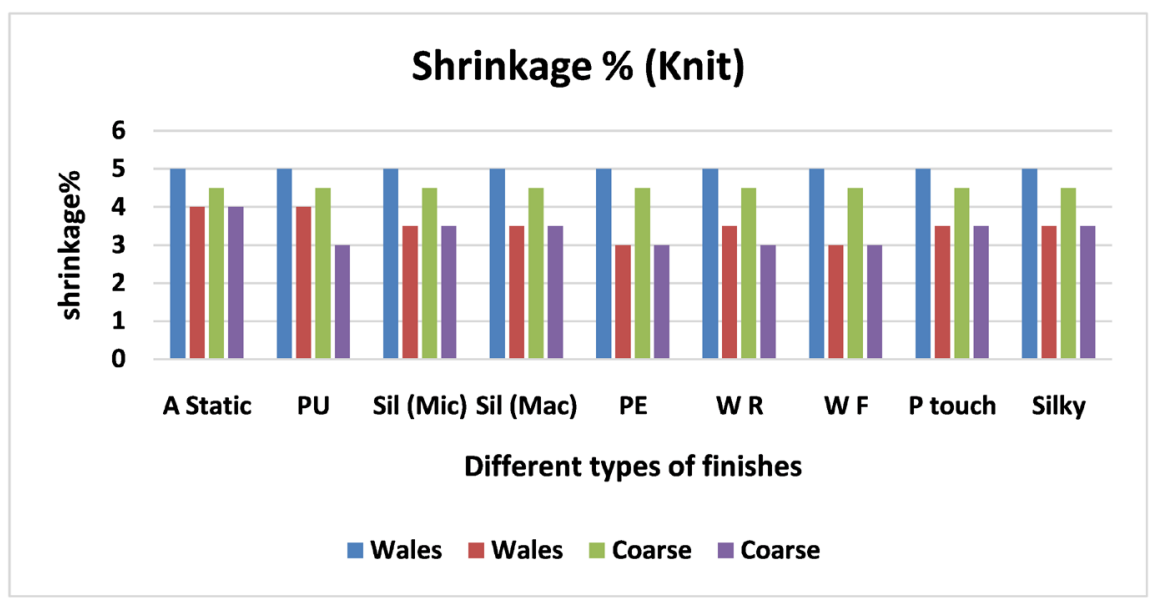

Figure 14. Shrinkage\% of single jersey (S/J) fabrics treated with different finishing agent.

Shrinkage percentage decreases after applying the finishes, which means the quality of fabric have improved.

\subsection{Water Absorbency Test (Spot Test)}

In a pipette a solution of $1 \%$ direct red (Congo red) is taken and droplet of solution put on the different places of the fabric. The time of the absorption of that water droplet on the fabric is observed. From below Table 7 water absorbing time in second is expressed for woven fabric after different finishes and Table 8 is given for $\mathrm{S} / \mathrm{J}$ fabric after different finishes.

The water absorbing time of original cotton was instant because of its hydrophilic property. The cellulose hydroxyl groups of cottons of cotton fabric make the water droplets spread instantly when it is placed in the surface of the fabric. After different finishing process the absorbing time is increased and it is reasonable as after soft finishing with both Sil (Mac) and Sil (Mic) softener and silky soft finishes, a waxy thin layer of silicon was made onto the fabric which prolong 
Table 7. Absorbency of woven fabric treated with different finishing agent.

\begin{tabular}{ccc}
\hline \multirow{2}{*}{ Name of the Finish } & \multicolumn{2}{c}{ Time in Second } \\
\cline { 2 - 3 } & Before Finish & After Finish \\
\hline Anti-Static & 15 & 20 \\
Polyurethane & 15 & 77 \\
Silicon (Micro) & 15 & 18 \\
Silicon (Macro) & 15 & 17 \\
Polyethylene & 15 & 75 \\
Water Repellent & 15 & - \\
Wrinkle Free & 15 & 13 \\
Paper Touch & 15 & 19 \\
Silky Soft & 15 & 80
\end{tabular}

Table 8. Absorbency of single jersey (S/J) fabric treated with different finishing agent.

\begin{tabular}{ccc}
\hline \multirow{2}{*}{ Name of the Finish } & \multicolumn{2}{c}{ Time in Second } \\
\cline { 2 - 3 } & Before Finish & After Finish \\
\hline Anti-Static & 12 & 17 \\
Polyurethane & 12 & 63 \\
Silicon (Micro) & 12 & 17 \\
Silicon (Macro) & 12 & 15 \\
Polyethylene & 12 & 75 \\
Water Repellent & 12 & - \\
Wrinkle Free & 12 & 10 \\
Paper Touch & 12 & 18 \\
Silky Soft & 12 & 60 \\
\hline
\end{tabular}

absorbing time. Whereas after antistatic finish, water absorbing time is not increased significantly as siligen Softener SIH has hydrophilic nature. After wrinkle free finish, absorbing time is reduced as it make fabric stiff so more friction is occurred between water droplet and fabric. It is obvious that after water repellent finishes, absorbing time will increases because of the hydrophobic nature of fluorocarbon. After PE finishing, it imparts a fuller, smoother handle to both woven and knit fabrics as it acts as a polyethylene dispersion. After PU finish, polyurethane dispersion make a film forming result in a fuller handle thus reduce absorbing time.

\subsection{Crease Recovery Angle}

It is done according to AATCC 66-2003 method and is taken the average values of warp and weft results. Table 9 and Figure 15 shows the crease recovery angle of woven fabric after different finishes and Table 10 and Figure 16 shows the crease recovery angle of knit fabrics which demonstrates that after wrinkle free finish, crease recovery angle is significantly increased for both woven and knit fabrics. 
Table 9. Crease recovery angle of woven fabric treated with different finishing agent.

\begin{tabular}{ccc}
\hline \multirow{2}{*}{ Name of the Finish } & \multicolumn{2}{c}{ Crease Recovery in Degrees } \\
\cline { 2 - 3 } & Before Finish & After Finish \\
\hline Anti-Static & 55 & 63 \\
Polyurethane & 55 & 69 \\
Silicon (Micro) & 55 & 70 \\
Silicon (Macro) & 55 & 71 \\
Polyethylene & 55 & 72 \\
Water Repellent & 55 & 67 \\
Wrinkle Free & 55 & 93 \\
Paper Touch & 55 & 75 \\
Silky Soft & 55 & 68 \\
\hline
\end{tabular}

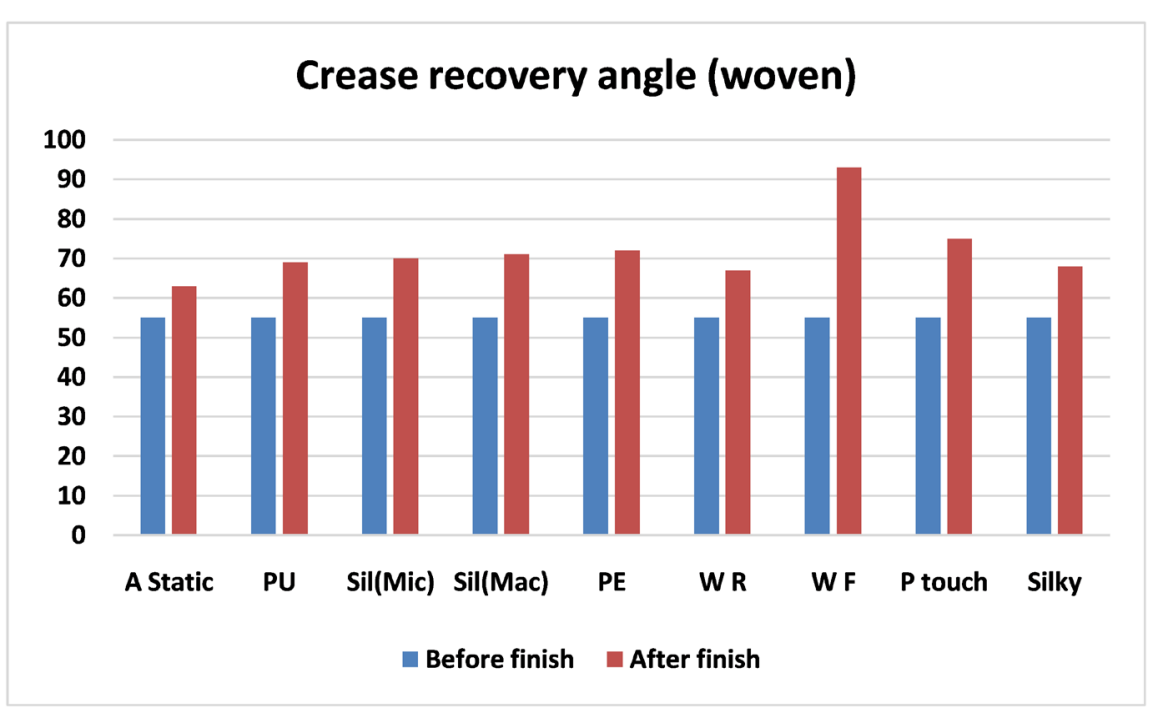

Figure 15. Crease recovery angle of woven fabric treated with different finishing agent.

Table 10. Crease recovery angle of single jersey (S/J) fabric treated with different finishing agent.

\begin{tabular}{ccc}
\hline \multirow{2}{*}{ Name of the Finish } & \multicolumn{2}{c}{ Crease Recovery Angle (S/J) } \\
\cline { 2 - 3 } & Before Finish & After Finish \\
\hline Anti-Static & 60 & 69 \\
Polyurethane & 60 & 72 \\
Silicon (Micro) & 60 & 70 \\
Silicon (Macro) & 60 & 75 \\
Polyethylene & 60 & 78 \\
Water Repellent & 60 & 77 \\
Wrinkle Free & 60 & 97 \\
Paper Touch & 60 & 75 \\
Silky Soft & 60 & 70 \\
\hline
\end{tabular}




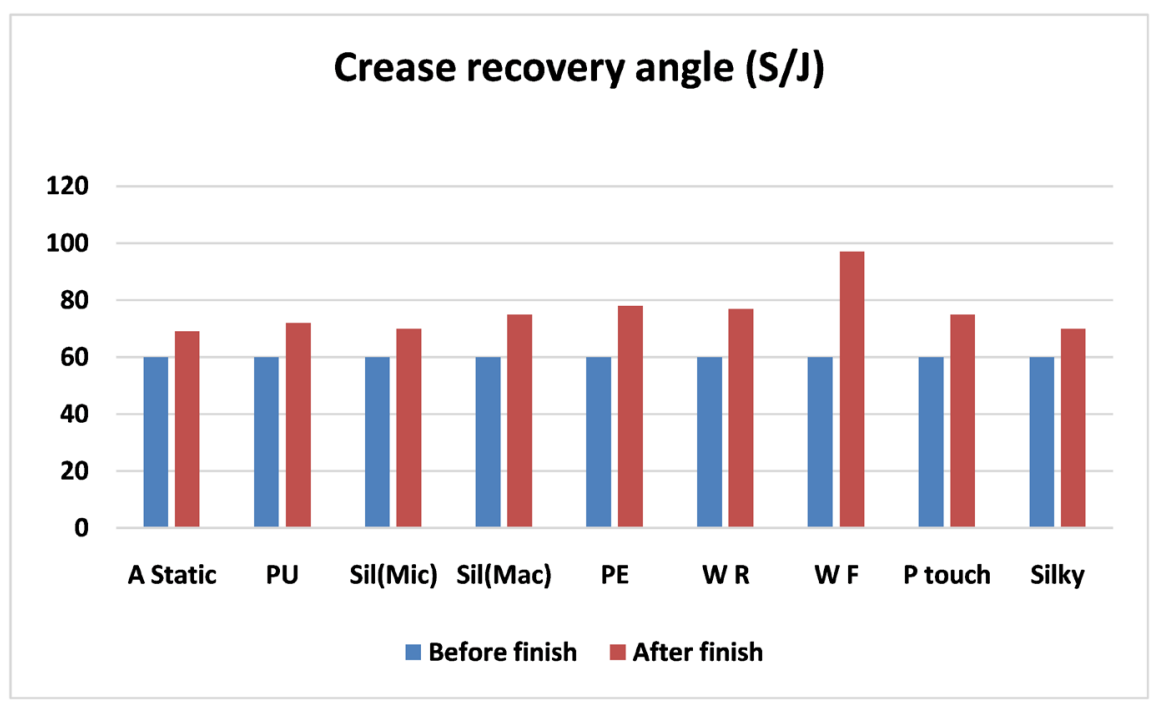

Figure 16. Crease recovery angle of single jersey $(\mathrm{S} / \mathrm{J})$ fabric treated with different finishing agent.

Table 11. Spray rating of both woven and knit $(\mathrm{S} / \mathrm{J})$ fabrics treated with water repellent chemical at $30 \mathrm{~g} / \mathrm{L}$.

\begin{tabular}{cccc}
\hline \multicolumn{3}{c}{ Spray rating (before wash) } \\
\hline Concentration $(\mathrm{g} / \mathrm{L})$ & Water repellent chemicals & Fabric & Rating \\
\hline & & Unfinished woven & 0 \\
$30 \mathrm{~g} / \mathrm{L}$ & Fluorocarbon & Finished woven & 90 \\
& & Unfinished knit (S/J) & 0 \\
& & Finished knit (S/J) & 90 \\
\hline
\end{tabular}

\subsection{Spray Rating Test}

Water repellent finish ( $\mathrm{W} \mathrm{R}$ ) is done by fluorocarbon based water repellent chemical in $30 \mathrm{~g} / \mathrm{L}$ concentration and is applied on woven and S/J knit dyed fabrics. There treated fabrics were evaluated by using AATCC 22-2001 method. Water repellency of both woven and knit (S/J) fabrics treated with fluorocarbon resin is shown in Table 11. Schindler and Hauser described that by completing the pad-dry-cure process, the heat treatment changes perfluoro side chains to almost crystalline structures to achieve optimal water repellency [11]. Compared with the untreated fabric, the finished fabrics had good water repellency with 90 grades.

\section{Conclusion}

The effects of special finishes were observed on both woven and knit 100\% cotton fabrics. To do so, 54 treated fabrics samples were tested and obtained results were evaluated. Different physical test results showed that different special finishes significantly are influenced on both woven and knit fabrics. This study mainly focused on the impact of chemical finishes, i.e., soft finish, silky soft 
finish, anti-static finish, water repellent finish, wrinkle free finish, paper touch finish, polyethylene finish and polyurethane, on the functional properties of cotton woven and knit fabrics. There was no remarkable deviation which is observed in GSM on the basis of different finishes. The obtained results show that the dimensional stability GSM is improved via chemical finishing; the wettability is decreased more via water repellent finish. Different chemical finishes did not cause significant change to bursting strength of knit fabrics and tensile strength for woven fabrics except soft finishes, which is another important conclusion of this work.

\section{Acknowledgements}

I would also like to express my deep gratitude to MICRO FIBRE for providing the lab facilities during the work. I would also like to thank Essential Clothing Ltd for providing knit fabrics. Again special gratitude also goes to the Wet Processing Lab \& the TTQC Lab of Bangladesh University of Textiles (Butex) for most of the physical tests.

\section{References}

[1] Schindler, W.D. and Hauser, P.J. (2004) Chemical Finishing of Textiles. Woodhead Publishing Ltd., Cambridge, 80-82.

[2] Kissa, E. (1984) Repellent Finishes. In: Lewin, M. and Sello, S.B., Eds., Handbook of Fibre Science and Technology, Vol. II. Functional Finishes, Pt. B. Chemical Processing of fibres and Fabrics, Ch. 2, Marcel Dekker, New York, 159-172.

[3] Ibrahim, N.A., El-Hossamy, M.E., Hassan, M.M., Refai, R. and Eid, B.M. (2008) Novel Pre-treatment Process to Promote Linen-Containing Fabrics Properties. Carbohydrate Polymers, 74, 881-891. https://doi.org/10.1016/j.carbpol.2008.05.009

[4] Xu, C.H., Jia, S.T., Zhang, J. and Ma, J.Z. (2010) Large-Area Fabrication of Superhydrophobic Surfaces for Practical Applications: An Overview. Science and Technology of Advanced Materials, 11, 1-15.

[5] Vishwanathan, N. (2004) Anti-Shrink/Anti-Stretch Treatment on Cellulosic Knits. Colourage, 50, 55-58.

[6] Castelvetro, V., Francini, G., Ciardelli, G. and Ceccato, M. (2001) Evaluating Fluorinated Acrylic Lattices as Textile Water and Oil Repellent Finishes. Textile Research Journal, 71, 399-406. https://doi.org/10.1177/004051750107100506

[7] Shao, H., Sun, J.Y., Meng, W.D. and Qing, F.L. (2004) Water and Oil Repellent and Durable Press Finishes for Cotton Based on a Perfluoroalkyl-Containing Multi-Epoxy Compound and Citric Acid. Textile Research Journal, 74, 851-855. https://doi.org/10.1177/004051750407401002

[8] Lee, H.J. and Michielsen, S. (2007) Preparation of a Superhydrophobic Rough surface. Journal of Polymer Science Part B: Polymer Physics, 45, 253-261. https://doi.org/10.1002/polb.21036

[9] Li, Z.R., Fu, K.J., Wang, L.J. and Liu, F. (2008) Synthesis of a Novel Perfluorinated Acrylate Copolymer Containing Hydroxyethyl Sulfone as Crosslinking Group and Its Application on Cotton Fabrics. Journal of Materials Processing Technology, 205, 243-248. https://doi.org/10.1016/j.jmatprotec.2007.11.284

[10] Roe, B. and Zhang, X. (2009) Durable hydrophobic Textile Fabric Finishing Using 
Silica Nanoparticles and Mixed Silanes. Textile Research Journal, 9, 1115-1122. https://doi.org/10.1177/0040517508100184

[11] Mohsin, M., Sarwar, N., Ahmed, S., Rasheed, A., Ahmad, F., Afzal, A. and Zafar, S. (2016) Maleic Acid Crosslinking of C-6 Fluorocarbon as Oil and Water Repellent Finish on Cellulosic Fabric. Journal of Cleaner Production, 112, 3525-3530. https://doi.org/10.1016/j.jclepro.2015.10.045

[12] Harifi, T. and Montazer, M. (2012) Past, Present and Future Prospects of Cotton Crosslinking: New Insight into Nano Particles. Carbohydrate Polymers, 88, 1125-1140. https://doi.org/10.1016/j.carbpol.2012.02.017

[13] Petersen, H. (1987) The Chemistry of Crease-Resist Crosslinking Agent. Review of Progress in Coloration and Related Topics, 17, 7-22. https://doi.org/10.1111/j.1478-4408.1987.tb03747.x

[14] Xu, W. and Wang, X. (2012) Understanding and Improving the Durability of Textiles. In: Woodhead Publishing Series in Textiles, Vol. 132, Woodhead Publishing Limited, Cambridge, UK.

[15] Hashem, M., Ibrahim, N.A., El-Shafei, A., Refaie, R. and Hauser, P. (2009) An Eco-Friendly-Novel Approach for Attaining Wrinkle-Free/Soft-Hand Cotton Fabric. Carbohydrate Polymers, 78, 690-703. https://doi.org/10.1016/j.carbpol.2009.06.004

[16] Shenai, V.A. (1994) Fabric Creasing and Its Control. Textile Dyer and Printer, 27, 18-29.

[17] Yang, C.Q., Wei, W.S. and Lickfield, G.C. (2000) Mechanical Strength of Durable Press Finished Cotton Fabric, Part II: Comparison of Crosslinking Agents with Different Molecular Structures and Reactivity. Textile Research Journal, 70, 143-147. https://doi.org/10.1177/004051750007000209

[18] Huang, K.S., Yang, K.L., Lin, S.J. and Lian, W.T. (2007) Antiwrinkle Treatment of Cotton Fabric with a Mixed Sol of TEOS-TTB/DMDHEU. Journal of Applied Polymer Science, 106, 2559-2564. https://doi.org/10.1002/app.25281

[19] Carty, P. and Byrne, M.S. (1987) Chemical Finishing of Natural Fibres. The Chemical and Mechanical Finishing of Textile Materials. 2nd Edition, Newcastle Upon Tyne Polytechnic Products Ltd., Newcastle, 32-49.

[20] Sahin, B. (1996) Fluorochemicals in Textile Finishing. International Textile Bulletin-Dyeing/Printing/Finishing, 42, 26-30.

[21] Grottenmuller, R. (1998) Fluorocarbons-An Innovative Aid to the Finishing of Textiles. Melliand International, 4, 278-281.

[22] Qi, H., Sui, K., Ma, Z., Wang, D., Sun, X. and Lu, J. (2002) Polymeric Fluorocarbon Coated Polyester Substrates for Waterproof Breathable Fabrics. Textile Research Journal, 72, 93-97. https://doi.org/10.1177/004051750207200201 